\title{
Focused ultrasound triggered release of tyrosine kinase inhibitor from thermosensitve liposomes for treatment of renal cell carcinoma
}

\author{
Caleb Abshire ${ }^{\mathrm{a} \dagger}$, Hakm Y. Murad ${ }^{\mathrm{b}, \mathrm{c} \dagger}$, Jaspreet S. Arora ${ }^{\mathrm{d}, \mathrm{e}}$, James Liu ${ }^{\mathrm{a}}$, Sree Harsha Mandava ${ }^{\mathrm{a}}$, Vijay T. \\ $\mathrm{John}^{\mathrm{d}, \mathrm{e}}$, Damir B. Khismatullin ${ }^{\mathrm{b}, \mathrm{f}, \mathrm{f}}$, and Benjamin R Lee ${ }^{\mathrm{a}, \mathrm{f}}$. \\ ${ }^{a}$ Department of Urology, Tulane University School of Medicine, 1430 Tulane Avenue, New Orleans, LA \\ 70112, USA \\ ${ }^{\mathrm{b}}$ Department of Biomedical Engineering, School of Science and Engineering, Tulane University, 6823 St. \\ Charles Avenue, New Orleans, LA 70118, USA \\ ${ }^{c}$ Tulane Institute for Integrative Engineering for Health and Medicine, Tulane University, 1324 Tulane \\ Avenue, New Orleans, LA 70112, USA \\ ${ }^{\mathrm{d}}$ Department of Chemical and Bimolecular Engineering, School of Science and Engineering, Tulane \\ University, 6823 St. Charles Avenue, New Orleans, LA 70118, USA \\ ${ }^{\mathrm{e}}$ Vector-Borne Infectious Disease Research Center, Tulane University, 1324 Tulane Avenue, New Orleans, \\ LA 70112, USA \\ ${ }_{\mathrm{f}}^{\mathrm{f}}$ Tulane Cancer Center, Tulane University School of Medicine, 1430 Tulane Avenue, New Orleans, LA \\ 70112, USA
}

$\dagger$ These authors contributed equally to this work

Corresponding author:

Benjamin R. Lee, MD

Professor of Urology \& Oncology

Tulane University School of Medicine

1430 Tulane Ave, SL42, Room 3522

New Orleans, LA 70112

Tel: 5049882985

Fax: 5049885059

Email: brlee@tulane.edu

Word count: 3,384

Abstract: 149

Figures: 8

References: 44

This work was supported by the Tulane University School of Medicine Endourology and Nanotechnology Research Fund (B.R.L.), National Science Foundation grant 1438537 (D.B.K.), American Heart Association grant 13 GRNT17200013 (D.B.K.), Louisiana Board of Regents grant LEQSF-EPS(2012)PFUND-292 (D.B.K.), and Louisiana Board of Regents grant LEQSF-EPS(2015)-PFUND-411 (V.T.J.). H.Y.M. gratefully acknowledges grant support from Newcomb-Tulane College and the Center for Engaged Learning and Teaching of Tulane University. 


\section{ABSTRACT}

This study reports, for the first time, development of tyrosine kinase inhibitor-loaded, thermosensitive liposomes (TKI/TSLs) and their efficacy for treatment of renal cell carcinoma when triggered by focused ultrasound (FUS). Uptake of these nanoparticles into renal cancer cells was visualized with confocal and fluorescent imaging of rhodamine B-loaded liposomes. The combination of TKI/TSLs and focused ultrasound was tested in an in vitro tumor model of renal cell carcinoma. According to MTT cytotoxic assay and flow cytometric analysis the combined treatment led to the least viability $(23.4 \pm 2.49 \%$, $\mathrm{p}<0.001)$, significantly lower than that observed from treatment with FUS $(97.6 \pm 0.67 \%$, n.s. $)$ or TKI/TSL $(71.0 \pm 3.65 \%, p<0.001)$ at 96 hours compared to control. The importance of this unique, synergistic combination was demonstrated in viability experiments with non-thermosensitive liposomes (TKI/NTSL+FUS: $58.8 \pm 1.5 \%$ vs TKI/TSL+FUS: $36.2 \pm 1.4 \%, \mathrm{p}<0.001$ ) and heated water immersion (TKI/TSL+WB43 ${ }^{\circ}: 59.3 \pm$ $2.91 \%$ vs TKI/TSL+FUS: $36.4 \pm 1.55 \%$, p <0.001). Our findings coupled with the existing use of focused ultrasound in clinical practice make the proposed combination of targeted chemotherapy, nanotechnology, and focused ultrasound a promising platform for enhanced drug delivery and cancer treatment.

Keywords: focused ultrasound, nanoparticles, renal cell carcinoma, thermally triggered chemotherapy, Tyrosine Kinase Inhibitor

\section{BACKGROUND}

Since FDA approval in 2005, tyrosine kinase inhibitors (TKI) have become the frontline medical approach for the treatment of advanced renal cell carcinoma (RCC). ${ }^{1,2}$ However, these recent advancements have not been without limitations. TKIs have poor bioavailability ${ }^{3}$ and lack tumor selectivity. Therefore, therapeutic drug dosing leads to systemic toxicity potentially manifested as hypothyroidism ${ }^{4,5}$, hypertension ${ }^{6-8}$, hand-foot syndrome ${ }^{7,8}$, gastrointestinal events ${ }^{9}$, and anorexia ${ }^{10}$, among others. These side effects not only negatively impact patient lives, but may also result in drug dosage reduction or interruption of treatment schedules, ultimately affecting patient outcomes and reducing overall survival. ${ }^{11,12}$

Nanotechnology is a powerful platform for augmenting traditional and targeted chemotherapy. Since the introduction of Doxil in $1995^{13}$, there has been growing interest in 
developing nanocarriers (liposomes, micelles, carbon nanotubes, and dendrimers) for chemotherapeutic drugs. ${ }^{14,15}$ Clinical trials of early liposomal drug formulations showed considerable success in the treatment of breast ${ }^{16}$, prostate $^{17}$, and ovarian ${ }^{18}$ cancers. These nanoparticles take advantage of the enhanced permeability and retention effect, which explains the increased accumulation of serum particles ranging in size from $100 \mathrm{~nm}$ to $150 \mathrm{~nm}$ at tumor sites $^{19,20}$. However, these first generation nanoparticles lack capability for specificity and controlled drug release ${ }^{14}$. Current research is directed at designing carriers with conjugated ligands to improve tumor selectivity ${ }^{21}$, carriers incorporating multiple payloads for thermal coupling $^{22,23}$, carriers with more clinically current antineoplastic agents ${ }^{24}$, and carriers capable of triggered drug release ${ }^{25-27}$.

High Intensity Focused Ultrasound (HIFU), a non-invasive means of tissue ablation, recently gained FDA approval for the treatment of prostate cance ${ }^{26}$ with clinical trials for renal cancer underway. ${ }^{28}$ Similar to diagnostic ultrasound, HIFU generates acoustic waves outside the body that propagate through a coupling medium and targeted to a specific organ such as prostate cancer. ${ }^{29}$ HIFU differs from diagnostic ultrasound in that acoustic waves converge to a focal point inside the body where constructive interference of waves occurs and absorption of acoustic energy leads to tissue heating. ${ }^{30}$ Nanocarriers, specifically thermosensitive liposomes (TSLs), can be strategically designed for triggered activation by focused ultrasound (FUS) and thereby have more directed cytotoxicity. ${ }^{15}$ Heat triggered TSLs have been shown to increase the cytotoxicity of doxorubicin $^{31,32}$; however, to date, no work has been published regarding TSLs loaded with a molecular pathway-targeting chemotherapeutic drug such as a TKI for treatment of RCC. We investigate the therapeutic potential of FUS combined with TKI (i.e. Sorafenib)-loaded TSLs in vitro. Specifically, this work tests the hypothesis that FUS-induced controlled release of TKI from TSLs will result in much lower cell viabilities than chemotherapeutic agent alone. RCC treated with FUS, non-thermosensitive liposomes (NTSLs), TSLs, a combination of FUS and NTSLs, or a combination of FUS and TSLs are analyzed for viability and liposomal uptake. In this paper, we demonstrate the advantage of combining multiple contemporary oncologic modalities to synergistically reduce cell viability without increasing therapeutic dose for high-risk RCC.

\section{METHODS}

\section{Materials \& Reagents}


Sorafenib (Nexovar) was obtained from Cayman Chemical (Ann Arbor, MI). 1,2-dipalmitoyl-snglycero-3-phosphocholine (DPPC) and hydrogenated soy L- $\alpha$-phosphatidylcholine (HSPC) were obtained from Avanti Polar Lipids (Alabaster, AL). Rhodamine B and cholesterol were obtained from Sigma Aldrich (St. Louis, MO).

\section{Nanoparticle Formulation}

Liposomes were formulated using thin film hydration technique following a previously published protocol. ${ }^{24}$ For TSLs, DPPC, HSPC, and cholesterol at molar ratio of 55.6:27.8:16.6 were dissolved in $10 \mathrm{~mL}$ of 2:1 v/v chloroform:methanol solution to prepare traditional TSLs with a glass transition temperature of $42-45^{\circ} \mathrm{C} .{ }^{33-39}$ For TKI/TSLs, $0.5 \mathrm{mg}$ of sorafenib was added to the solution of the lipids and was evaporated at $100 \mathrm{mbar}, 125 \mathrm{rpm}$ at room temperature for $2.5 \mathrm{~h}$ using a Buchi Rotary Evaporator (Buchi, New Castle, DE). The pressure was decreased to 0 mbar and the mixture was rotated at $125 \mathrm{rpm}$ overnight for complete evaporation of solvent to create a thin lipid film. The dry lipid film was hydrated with $5 \mathrm{ml}$ of PBS buffer (10 mM, pH 7.4) and the mixture was rotated at $125 \mathrm{rpm}$ at $55^{\circ} \mathrm{C}$ in a water bath for $1 \mathrm{~h}$. Liposomes were then extruded through a mini-extruder (Avanti Polar Lipids) at $55^{\circ} \mathrm{C}$ using a $100 \mathrm{~nm}$ membrane (Whatman Nuclepore Track-etch membrane, Sigma Aldrich). Control liposomes without a drug were prepared without the addition of sorafenib. Rhodamine B loaded fluorescent liposomes were formulated using a similar protocol without the addition of sorafenib. Instead, $5 \mathrm{~mL}$ of $1 \mathrm{mg} / 1 \mathrm{~mL}$ solution of rhodamine B in PBS (10 mM, pH 7.4) was added during the hydration step. In the final step, the extruded solution was passed through a gel column (PD-10 Desalting Columns, GE Healthcare, Piscataway, NJ) to separate the unencapsulated species (dye or drug) from the liposomes. Since the TKI is not soluble in water, most of it is encapsulated in the bilayer during hydration and extrusion resulting in an almost 100\% encapsulation efficiency. NTSLs were formulated using a similar protocol as TSLs with the notable exclusion of HSPC and cholesterol. Instead, $100 \mathrm{mg}$ of DPPC was dissolved in $10 \mathrm{~mL}$ of 2:1 chloroform:methanol solution. $20 \mu \mathrm{L}$ of $25 \mathrm{mg} / \mathrm{mL}$ of sorafenib in DMSO was also added to solution to a total of $0.5 \mathrm{mg}$ sorafenib. All other steps followed protocol as stated above.

\section{Nanoparticle Characterization}

Liposomes were characterized by cryogenic transmission electron microscopy (cryo-TEM) (G2 F30 Tecnai, FEI, Hillsboro, OR) operated at $200 \mathrm{kV}$. Particle zeta potential and size were measured by the Zetasizer Nano ZS (Malvern Instruments, Malvern, UK). 
The calculation of the encapsulation efficiency was performed by quantifying the drug in $1 \mathrm{~mL}$ of liposomes and comparing it to the control liposomes without sorafenib. Liposomes were mixed with $4 \mathrm{ml}$ of methanol to break the vesicles. The concentration of sorafenib in the solution was then measured using the UV-1700 PharmSpec UV-Vis Spectrophotometer (Shimadzu, Kyoto, Japan) at a wavelength of $264.7 \mathrm{~nm}$ (maximum absorption of SF in mixture of PBS: methanol 1:4). The concentration of sorafenib was calculated using a calibration curve and percent encapsulation efficiency (\%EE) was calculated as follows:

$$
\% E E=\frac{\text { amount of drug in NP }}{\text { amount of initially added drug }} \times 100 \%
$$

\section{Cell Culture}

A human metastatic RCC cell line, clear cell type (786-0) was obtained from American Type Culture Collection (Manassas, VA) and cultured in RPMI 1640 medium supplemented with 10\% fetal bovine serum and 1\% Antibiotic-Antimycotic (Life Technologies, Grand Island, NY). Cells were cultured at $37^{\circ} \mathrm{C}$ with $5 \% \mathrm{CO}_{2}$ and $95 \%$ humidity. The culture medium was replaced every 2 days and cells were subcultured upon reaching optimal confluence. Renal tumor pellets were formed by centrifuging 2 million cells in $100 \mu \mathrm{L}$ of the medium within a $0.2 \mathrm{~mL}$ PCR tube (BioRad, Hercules, CA).

\section{Cellular Uptake}

Uptake of TSLs was confirmed by confocal laser microscopy (Nikon A1 ${ }^{+} /$Eclipse Ti, Nikon Instruments, Melville, NY). TSLs loaded with rhodamine B were incubated with RCC cells in a 6-well plate with glass well bottoms (MatTek, Ashland, MA) and incubated for 6 hours before imaging with a $20 \mathrm{X}$ objective.

\section{Focused Ultrasound}

A 1.1 MHz single-element, concave transducer (model H102, Sonic Concepts, Bothell, WA) with bandwidth from 0.748 to $1.380 \mathrm{MHz}$ and geometric focal length of $63.2 \mathrm{~mm}$ was used in all experiments. The transducer, housed in stainless steel with an active diameter of $64 \mathrm{~mm}$, was placed inside a $37^{\circ} \mathrm{C}$ degassed water chamber. A built-in arbitrary function generator (33220A, Agilent Technology, Santa Clara, CA) produced an input sinusoidal signal that passed through a fixed gain (50dB) power amplifier (ENL 2100L, Electronics \& Innovation, Rochester, NY) and then entered the transducer. The FUS signal strength was monitored using a 2 Giga-samples/s 
InfiniiVision DSO-X-2014A oscilloscope (Agilent Technology). Temperature measurements during FUS targeting were collected using a mini-hypodermic type $\mathrm{T}$ thermocouple of diameter $200 \mu \mathrm{m}$ and recorded by a 4-channel temperature meter (SDL200, Extech Instruments, Waltham, MA). During all experiments the FUS transducer was operated in continuous mode for 90 seconds at an acoustic output power of $2.73 \mathrm{~W}$, corresponding to spatial peak temporal average intensity $I_{S P T A}$ of $0.240 \mathrm{~kW} / \mathrm{cm}^{2}$, to ensure temperature rise to $43^{\circ} \mathrm{C}$.

\section{Treatment}

786-O renal cancer cells were pretreated with empty TSLs, free TKI, TKI/NTSLs, or TKI/TSLs 4 hours prior to tumor model formation. The TKI, Sorafenib, was administered at a concentration of $10 \mu \mathrm{M}$ in all experimental groups per previously established protocol ${ }^{38}$. Tumor models were then exposed to heat activation with FUS, water bath immersion, or no heat activation for 90 seconds.

\section{Cell Viability}

Viability was measured using MTT assay (Sigma-Aldrich) and Alexa Fluor ${ }^{\circledR} 488$ Annexin V/Dead Cell Apoptosis assay kit (Thermo Fisher Scientific, Waltham, MA). For MTT assay, treated cells were plated in $0.1 \mathrm{ml}$ 96-well flat bottom plates (Fischer Scientific, Pittsburgh, PA) and incubated for varying time points. Then, the cells were exposed to $20 \mu \mathrm{L}$ of $5 \mathrm{mg} / \mathrm{mL}$ MTT solution in PBS for $4 \mathrm{~h}$. This solution was then replaced with $170 \mu \mathrm{L}$ of DMSO to dissolve the formazan crystals and assayed at $540 \mathrm{~nm}$ using an absorbance plate reader (ELx808, BioTek Instruments, Winooski, VT). For apoptosis assay, cells from pellets were reseeded into T-75 tissue culture flasks with $10 \mathrm{~mL}$ of culture medium per flask. After incubation for different time points, viable, early apoptotic, late apoptotic/necrotic populations of cells were assessed by flow cytometry (Attune Acoustic Focusing Cytometer, Applied Biosystems, Grand Island, NY). The Annexin V/Dead Cell Apoptosis Kit stains apoptotic cells with Annexin V (green) and necrotic cells with both Propidium Iodide (PI, red) and Annexin V (green). Early apoptotic cells were identified as PI negative and Annexin V positive, whereas the cells that are PI and Annexin V positive were considered late apoptotic/necrotic. Cells not stained with Annexin V or PI were considered to be viable.

\section{Statistics}

All experiments were performed in triplicates. The results were evaluated with $t$-test and ANOVA using GraphPad Prism version 5.0.2 (GraphPad Software, La Jolla, CA). The statistical data were 
presented as mean \pm standard error of the mean (SEM). Statistically significant differences were set at $p<0.05$ (95\% confidence).

\section{RESULTS}

\section{Nanoparticle Characterization}

Cryo-TEM images of the TSLs with and without sorafenib loading are shown in Figure 1. Empty TSLs in Figure 1A are unilamellar with a particle diameter between 100-200 nm. Sorafenib loading in Figure 1B does not alter the morphology of the liposomes and these liposomes are also unilamellar with a particle diameter between 100-200 nm. Average diameter of the empty TSLs and TKI-loaded TSLs was measured by dynamic light scattering (DLS) as 103 and $107 \mathrm{~nm}$ with a Polydispersity Index (PDI) of 0.198 and 0.225 , respectively. The DLS results are in agreement with the cryo-TEM images. The \% EE of sorafenib in TKI-loaded TSLs was evaluated as $99.7 \pm$ $0.81 \%$. The zeta potential for the empty TSLs was $-2.14 \pm 5.32 \mathrm{mV}$ and TKI-loaded TSL was $2.39 \pm 1.84 \mathrm{mV}$. These zeta potential values suggest no significant surface charge exists on the liposomes, which is expected because of the neutral lipids used to formulate the liposomes.

\section{In Vitro Cellular Uptake}

Confocal laser microscopy of the cells incubated with rhodamine B-loaded TSLs was performed to confirm uptake of liposomes by cells. Red fluorescence from rhodamine B is seen within cells in Figure 2A, with bright field and red fluorescence signals merged into one image indicating the presence of rhodamine B inside cells and confirming nanoparticle uptake. In Figures 2B and 2C red fluorescence and bright field imaging signals are respectively presented. Confocal z-stack images were taken of the cells at different heights and were compiled into a 3-D image in Figure 2D. We observed multiple red flurorescent clusters spread throughout the cells, confirming the presence of rhodamine B-loaded liposomes in intracellular compartments.

\section{Cell Viability}

Individual Components of Combined Treatment: To evaluate the cellular toxicity of FUSactivated TKI/TSL's we first investigated the cellular effects of each individual component in the proposed combination treatment. We treated 786-O cells with empty TSLs, FUS alone, free TKI, empty TSLs with FUS exposure, free TKI with FUS exposure, and TKI/TSL's without FUS exposure. Cell viability was assessed at 24,48 , and 72 hours post treatment for all these groups 
(see figure 3A). At all three time points, cells treated with empty TSLs, FUS alone, TKI alone, or empty TSLs with FUS activation were not significantly different from untreated cells (control, $p$ $>0.05)$. Cells treated with free TKI and FUS activation showed no significant difference from control at 24 hours; however, at 48 and 72 hours there was a decrease in viability to $80.6 \pm 1.13 \%$ $(\mathrm{p}<0.001)$ and $79.3 \pm 6.49 \%(\mathrm{p}<0.001)$, respectively. The lowest viability observed was in the group of TKI/TSL treated cells $(83.7 \pm 2.33 \%, \mathrm{p}<0.01$ at 24 hours; $79.0 \pm 3.61 \%, \mathrm{p}<0.001$ at 48 hours, and $52.7 \pm 4.10 \%, \mathrm{p}<0.001$ at 72 hours).

Combined Treatment: Figure 3B shows that FUS-activated TKI/TSL's induced the largest amount of cellular death at $24,48,72$, and 96 hours post treatment, with viability reduced to 23.4 $\pm 2.49 \%$ ( $p<0.001)$ at 96 hours. Additionally, the FUS-activated TKI/TSL group but not the individual treatment groups (FUS, TKI, TKI/TSL) showed continually decreasing viability for each time point. Figure 3C illustrates data collected at 12 hour intervals over 4 days to better characterize the differing response of cells treated with TKI/TSL's alone and cells treated with TKI/TSL's and then activated by FUS. Both the TKI/TSL and TKI/TSL + FUS activation groups showed decreasing viability up until 72 hours post treatment. After 72 hours, the trend was reversed in cells treated with TKI/TSL's alone, with a statistically significant increase in viability between 72 and 96 hours $(61.7 \pm 1.78 \%$ vs. $71.0 \pm 3.65 \%$, p < 0.05$)$. However, this trend was not observed for cells treated with a combination of TKI/TSL's and FUS activation, i.e., their viability continuously decreased up to 96 hours post treatment.

Figure 4 compares the effect of FUS activation on both TKI-loaded non-thermosensitive liposomes (TKI/NTSLs) and TKI/TSLs. FUS activation of TKI/NTSLs showed no statistically significant difference from TKI/NTSLs without FUS activation at 24, 48, and 72 hours. However, statistically significant reductions in cell viability were observed when comparing TKI/TSLs without and with FUS at 48 hours $(79.0 \pm 3.61 \%$ vs $55.7 \pm 1.62 \%, \mathrm{p}<0.001)$ and 72 hours $(52.7 \pm 4.10 \%$ vs $36.2 \pm 1.37 \%, \mathrm{p}<0.001)$.

Figure 5 shows a comparison of viability for 786-O cells pretreated with TKI/TSLs and then immersed in a water bath at $43^{\circ} \mathrm{C}$ or activated with FUS. At 24,48 , and 72 hours, activation of TKI/TSLs with FUS greatly reduced cell viability, as compared to water bath immersion at equivalent temperature. For example, the viability of cells exposed to water bath immersion was $59.3 \pm 2.91 \%$ at 72 hours, which was statistically significant from the viability of cells exposed to FUS $(36.4 \pm 1.55 \%, \mathrm{p}<0.001)$. This result points out that FUS does not only activate TKI/TSLs but also makes the cells more susceptible to TKI. 


\section{Flow Cytometry}

Figure 6A-C displays the population distribution of viable, early apoptotic, and late apoptotic/necrotic cells as measured by flow cytometry at 72 hours post treatment with TKI alone, TKI/TSLs, and TKI/TSLs with FUS activation, respectively. The majority of cells treated with TKI alone were viable because they were negative for both Annexin V and PI. With cells exposed to TKI/TSLs, the population density began to shift from the bottom left corner (viable) to the bottom right and upper left corners (early apoptotic and late apoptotic/necrotic). A similar but more prominent shift in population density was seen in cells exposed to TKI/TSLs with FUS activation, indicating a larger percentage of apoptotic and necrotic cells. The total percentage of the viable cell population, as measured by flow cytometry, is shown for different treatments and time points in Figure 6D. There was a statistically significant difference in the population of viable cells in the TKI/TSLs and control groups at 72 hours $(60.7 \pm 4.7 \%$ vs. $86.3 \pm 0.60 \%$, p < 0.01). Likewise, a statistically significant decrease in viability was observed between TKI/TSLs with and without FUS activation at 72 hours $(39.5 \pm 8.85 \%$ vs. $60.7 \pm 4.7 \%, \mathrm{p}<0.01)$. This result is consistent with the MTT assay data.

\section{DISCUSSION}

In this study, we successfully created a TKI-loaded, thermosensitive liposomal nanoparticle capable of targeted drug release with FUS activation leading to increased tumor death (Figure 7). In vitro tumor pellets of RCC exposed to combined TKI/TSL with FUS treatment had much lower viability when compared to cells treated with individual components. Interestingly, cell viability reduction seen for each individual component was markedly less than the reduction in viability observed with combined treatment. This finding demonstrates existence of a synergistic relationship between TKI/TSLs and FUS. Figure 8 summarizes our data on cell viability and illustrates this synergy between TKI/TSL and FUS. RCC treatment with TKI/TSL and FUS showed approximately 2.5-fold higher cellular death in RCC than an additive effect of individual treatment arms.

Our experiments comparing TKI/NTSLs and TKI/TSLs highlight the importance of utilizing liposomes with thermally triggered drug release mechanisms, while our water bath immersion experiments indicate that the anti-cancer effect of FUS cannot be simply explained by FUS-induced tissue heating. In addition to thermal effects, FUS can weaken cancer cells by mechanical means, which makes its uniquely advantageous for activation and enhancement of 
TSL-based chemotherapy. In this study both temperature and non-temperature sensitive liposomes are nonselective in their distribution throughout the body with the exception of increased tumor accumulation due to the enhanced permeability and retention effect. Following cellular endocytosis, NTSLs and TSLs remain inactive until acidification within endosomes or lysosomes $^{40}$. The resulting liposomal bilayer destabilization initiates a time and $\mathrm{pH}$ dependent release of payload ${ }^{41}$. In order to selectively increase cytotoxicity at the tumor site, FUS is applied to activate accumulated TSLs in seconds, irrespective of cellular degradation pathways ${ }^{39}$. Once released from the TSL, sorafenib acts directly on membrane receptor tyrosine kinases (VEGF, PDGFR, RET, \& c-Kit) or on their downstream signal mediators, namely members of the Raf serine/threonine kinase family ${ }^{42}$. Inhibition of these pathways decreases angiogenesis and cellular proliferation while promoting apoptotic pathways leading to the cellular toxicity seen in this study. Additional FUS dependent treatment effects include acoustic streaming ${ }^{43}$ that can increase drug bioavailability ${ }^{44}$ and mechanical stimulation that can lead to alterations in gene expression and increase TKI sensitivity. For example, FUS is known to increase the expression of Fas ligand $(\mathrm{FasL})^{45,46}$ while Sorafenib has been shown to upregulate expression of the Fas death receptor (FasR). ${ }^{47}$ These two events result in enhancement of Fas mediated apoptosis and could explain the increased population of apoptotic cells seen in the combined TKI/TSL and FUS treatment group. These results are in line with our previous findings of FUS behaving synergistically with antineoplastic chemical agents, such as ethanol. ${ }^{43,48}$

While recent advancements in the development of targeted chemotherapeutics have drastically changed the clinical approach to RCC treatment, overall survival (OS) for patients remains relatively unchanged with subsequent generations of TKIs. ${ }^{49}$ However, as demonstrated in the PISCES trial, quality of life improvements seen in patients treated with newer TKIs have led to patient preference of the newer drugs. ${ }^{50}$ Our combined treatment shows markedly improved RCC cytotoxicity when compared to TKI treatment alone and implementation of this strategy would allow for equivalent RCC death with lower dosages of TKI. This combined treatment would not only benefit patients with RCC but potentially other patients currently on TKI therapy for other cancers such as colorectal cancer ${ }^{51}$, liver cancer ${ }^{52}$, and/or thyroid cancer ${ }^{52,53}$. The current use of FUS in clinical practice and the findings of our investigations make the combination of targeted therapy, nanotechnology, and focused ultrasound a promising platform for enhanced drug delivery and cancer treatment.

Acknowledgments: This work was supported by the Tulane University School of Medicine Endourology and Nanotechnology Research Fund (B.R.L.), National Science Foundation grant 
1438537 (D.B.K.), American Heart Association grant 13GRNT17200013 (D.B.K.), Louisiana Board of Regents grant LEQSF-EPS(2012)-PFUND-292 (D.B.K.), and Louisiana Board of Regents grant LEQSF-EPS(2015)-PFUND-411 (V.T.J.). H.Y.M. gratefully acknowledges grant support from Newcomb-Tulane College and the Center for Engaged Learning and Teaching of Tulane University. The authors thank Daishen Luo and Heng Yu for assistance with flow cytometric analysis and Stephen Ashe for help with liposomal formulation.

\section{REFERENCES}

1. Escudier B, Albiges L, Sonpavde G. Optimal management of metastatic renal cell carcinoma: current status. Drugs 2013; 73(5): 427-38.

2. U.S. Food and Drug Administration CfDEaR. Sorafenib NDA 21-923 approval letter. December 20, 2005.

http://www.accessdata.fda.gov/drugsatfda docs/nda/2005/021923 s000 Nexavar Approv.pdf (accessed October 13 2015).

3. Wang XQ, Fan JM, Liu YO, Zhao B, Jia ZR, Zhang Q. Bioavailability and pharmacokinetics of sorafenib suspension, nanoparticles and nanomatrix for oral administration to rat. Int J Pharm 2011; 419(1-2): 339-46.

4. Tamaskar I, Bukowski R, Elson P, et al. Thyroid function test abnormalities in patients with metastatic renal cell carcinoma treated with sorafenib. Ann Oncol 2008; 19(2): 265-8.

5. Torino F, Corsello SM, Longo R, Barnabei A, Gasparini G. Hypothyroidism related to tyrosine kinase inhibitors: an emerging toxic effect of targeted therapy. Nat Rev Clin Oncol 2009; 6(4): 219-28.

6. Kandula P, Agarwal R. Proteinuria and hypertension with tyrosine kinase inhibitors. Kidney Int 2011; 80(12): 1271-7.

7. Haddad H, Rini BI. Current treatment considerations in metastatic renal cell carcinoma. Curr Treat Options Oncol 2012; 13(2): 212-29.

8. Pham A, Ye DW, Pal S. Overview and management of toxicities associated with systemic therapies for advanced renal cell carcinoma. Urol Oncol 2015; 33(12): 517-27.

9. Santoni M, Conti A, De Giorgi U, et al. Risk of gastrointestinal events with sorafenib, sunitinib and pazopanib in patients with solid tumors: a systematic review and meta-analysis of clinical trials. Int J Cancer 2014; 135(4): 763-73.

10. Naito S, Tsukamoto T, Usami M, Fujimoto H, Akaza H. An early phase II trial of S-1 in Japanese patients with cytokine-refractory metastatic renal cell carcinoma. Cancer Chemother Pharmacol 2010; 66(6): 1065-70.

11. Ruiz JN, Belum VR, Creel P, Cohn A, Ewer M, Lacouture ME. Current practices in the management of adverse events associated with targeted therapies for advanced renal cell carcinoma: a national survey of oncologists. Clin Genitourin Cancer 2014; 12(5): 341-7.

12. Hayes SM, Bowser AD, Mortimer J, et al. Practice challenges affecting optimal care as identifed by US medical oncologists who treat renal cell carcinomas. J Community Support Oncol 2014; 12(6): 197-204.

13. Barenholz Y. Doxil(R)--the first FDA-approved nano-drug: lessons learned. $J$ Control Release 2012; 160(2): 117-34. 
14. Davis ME, Chen ZG, Shin DM. Nanoparticle therapeutics: an emerging treatment modality for cancer. Nat Rev Drug Discov 2008; 7(9): 771-82.

15. Ta T, Porter TM. Thermosensitive liposomes for localized delivery and triggered release of chemotherapy. J Control Release 2013; 169(1-2): 112-25.

16. Verma S, Dent S, Chow BJ, Rayson D, Safra T. Metastatic breast cancer: the role of pegylated liposomal doxorubicin after conventional anthracyclines. Cancer Treat Rev 2008; 34(5): 391-406.

17. Kroon J, Metselaar JM, Storm G, van der Pluijm G. Liposomal nanomedicines in the treatment of prostate cancer. Cancer Treat Rev 2014; 40(4): 578-84.

18. Gibson JM, Alzghari S, Ahn C, Trantham H, La-Beck NM. The role of pegylated liposomal doxorubicin in ovarian cancer: a meta-analysis of randomized clinical trials. Oncologist 2013; 18(9): 1022-31.

19. Matsumura Y, Maeda H. A new concept for macromolecular therapeutics in cancer chemotherapy: mechanism of tumoritropic accumulation of proteins and the antitumor agent smancs. Cancer Res 1986; 46(12 Pt 1): 6387-92.

20. Longmire M, Choyke PL, Kobayashi H. Clearance properties of nano-sized particles and molecules as imaging agents: considerations and caveats. Nanomedicine (Lond) 2008; 3(5): 703-17.

21. Matsumura Y, Gotoh M, Muro K, et al. Phase I and pharmacokinetic study of MCC-465, a doxorubicin (DXR) encapsulated in PEG immunoliposome, in patients with metastatic stomach cancer. Ann Oncol 2004; 15(3): 517-25.

22. Stern JM, Stanfield J, Kabbani W, Hsieh JT, Cadeddu JA. Selective prostate cancer thermal ablation with laser activated gold nanoshells. J Urol 2008; 179(2): 748-53.

23. Fisher JW, Sarkar S, Buchanan CF, et al. Photothermal response of human and murine cancer cells to multiwalled carbon nanotubes after laser irradiation. Cancer Res 2010; 70(23): 9855-64.

24. Liu J, Boonkaew B, Arora J, et al. Comparison of sorafenib-loaded poly (lactic/glycolic) acid and DPPC liposome nanoparticles in the in vitro treatment of renal cell carcinoma. J Pharm Sci 2015; 104(3): 1187-96.

25. Schroeder A, Honen R, Turjeman K, Gabizon A, Kost J, Barenholz Y. Ultrasound triggered release of cisplatin from liposomes in murine tumors. J Control Release 2009; 137(1): 63-8.

26. Zhang Q, Tang J, Fu L, et al. A pH-responsive alpha-helical cell penetrating peptide-mediated liposomal delivery system. Biomaterials 2013; 34(32): 7980-93.

27. Lu J, Choi E, Tamanoi F, Zink JI. Light-activated nanoimpeller-controlled drug release in cancer cells. Small 2008; 4(4): 421-6.

28. Ritchie RW, Leslie T, Phillips R, et al. Extracorporeal high intensity focused ultrasound for renal tumours: a 3-year follow-up. BJU Int 2010; 106(7): 1004-9. 29. Kaproth-Joslin KA, Nicola R, Dogra VS. The History of US: From Bats and Boats to the Bedside and Beyond: RSNA Centennial Article. Radiographics 2015; 35(3): 960-70.

30. Maloney E, Hwang JH. Emerging HIFU applications in cancer therapy. Int J Hyperthermia 2015; 31(3): 302-9. 
31. Dromi S, Frenkel V, Luk A, et al. Pulsed-high intensity focused ultrasound and low temperature-sensitive liposomes for enhanced targeted drug delivery and antitumor effect. Clin Cancer Res 2007; 13(9): 2722-7.

32. Al-Ahmady ZS, Al-Jamal WT, Bossche JV, et al. Lipid-peptide vesicle nanoscale hybrids for triggered drug release by mild hyperthermia in vitro and in vivo. ACS Nano 2012; 6(10): 9335-46.

33. de Smet M, Langereis S, van den Bosch S, Grull H. Temperature-sensitive liposomes for doxorubicin delivery under MRI guidance. J Control Release 2010; 143(1): 120-7.

34. Al-Ahmady ZS, Chaloin 0, Kostarelos K. Monoclonal antibody-targeted, temperature-sensitive liposomes: in vivo tumor chemotherapeutics in combination with mild hyperthermia. J Control Release 2014; 196: 332-43.

35. Al-Ahmady ZS, Scudamore CL, Kostarelos K. Triggered doxorubicin release in solid tumors from thermosensitive liposome-peptide hybrids: Critical parameters and therapeutic efficacy. Int J Cancer 2015; 137(3): 731-43.

36. Needham D, Anyarambhatla G, Kong G, Dewhirst MW. A new temperaturesensitive liposome for use with mild hyperthermia: characterization and testing in a human tumor xenograft model. Cancer Res 2000; 60(5): 1197-201.

37. de Smet M, Heijman E, Langereis S, Hijnen NM, Grull H. Magnetic resonance imaging of high intensity focused ultrasound mediated drug delivery from temperature-sensitive liposomes: an in vivo proof-of-concept study. J Control Release 2011; 150(1): 102-10.

38. Arora JS, Murad HY, Ashe S, et al. Ablative Focused Ultrasound Synergistically Enhances Thermally Triggered Chemotherapy for Prostate Cancer in Vitro. Mol Pharm 2016; 13(9): 3080-90.

39. Ta T, Bartolak-Suki E, Park EJ, Karrobi K, McDannold NJ, Porter TM. Localized delivery of doxorubicin in vivo from polymer-modified thermosensitive liposomes with MR-guided focused ultrasound-mediated heating. J Control Release 2014; 194: 71-81.

40. Pollock S, Antrobus R, Newton L, et al. Uptake and trafficking of liposomes to the endoplasmic reticulum. FASEB J 2010; 24(6): 1866-78.

41. Huth US, Schubert R, Peschka-Suss R. Investigating the uptake and intracellular fate of $\mathrm{pH}$-sensitive liposomes by flow cytometry and spectral bioimaging. J Control Release 2006; 110(3): 490-504.

42. Wilhelm S, Carter C, Lynch M, et al. Discovery and development of sorafenib: a multikinase inhibitor for treating cancer. Nat Rev Drug Discov 2006; 5(10): 835-44. 43. Hoang NH, Murad HY, Ratnayaka SH, Chen C, Khismatullin DB. Synergistic ablation of liver tissue and liver cancer cells with high-intensity focused ultrasound and ethanol. Ultrasound Med Biol 2014; 40(8): 1869-81.

44. Sutton JT, Haworth KJ, Pyne-Geithman G, Holland CK. Ultrasound-mediated drug delivery for cardiovascular disease. Expert Opin Drug Deliv 2013; 10(5): 57392.

45. Lu P, Zhu XQ, Xu ZL, Zhou Q, Zhang J, Wu F. Increased infiltration of activated tumor-infiltrating lymphocytes after high intensity focused ultrasound ablation of human breast cancer. Surgery 2009; 145(3): 286-93. 
46. Liu Y, Kon T, Li C, Zhong P. High intensity focused ultrasound-induced gene activation in sublethally injured tumor cells in vitro. J Acoust Soc Am 2005; 118(5): 3328-36.

47. Park MA, Reinehr R, Haussinger D, et al. Sorafenib activates CD95 and promotes autophagy and cell death via Src family kinases in gastrointestinal tumor cells. Mol Cancer Ther 2010; 9(8): 2220-31.

48. Chen C, Liu Y, Maruvada S, Myers M, Khismatullin D. Effect of ethanol injection on cavitation and heating of tissues exposed to high-intensity focused ultrasound. Phys Med Biol 2012; 57(4): 937-61.

49. Motzer RJ, Hutson TE, Cella D, et al. Pazopanib versus sunitinib in metastatic renal-cell carcinoma. $N$ Engl J Med 2013; 369(8): 722-31.

50. Escudier B, Porta C, Bono P, et al. Randomized, controlled, double-blind, cross-over trial assessing treatment preference for pazopanib versus sunitinib in patients with metastatic renal cell carcinoma: PISCES Study. J Clin Oncol 2014; 32(14): 1412-8.

51. Grothey A, Van Cutsem E, Sobrero A, et al. Regorafenib monotherapy for previously treated metastatic colorectal cancer (CORRECT): an international, multicentre, randomised, placebo-controlled, phase 3 trial. Lancet 2013; 381(9863): 303-12.

52. Llovet JM, Ricci S, Mazzaferro V, et al. Sorafenib in advanced hepatocellular carcinoma. $N$ Engl J Med 2008; 359(4): 378-90.

53. Klein Hesselink EN, Steenvoorden D, Kapiteijn E, et al. Therapy of endocrine disease: response and toxicity of small-molecule tyrosine kinase inhibitors in patients with thyroid carcinoma: a systematic review and meta-analysis. Eur J Endocrinol 2015; 172(5): R215-25.

Figure Legends

\section{Figure 1}

Cryo-TEM images of thermosensitive liposomes. Figure 1A: free liposomes, without drug; figure 1B: TKI-loaded liposomes. Equivalent and intact surface morphology for both particles illustrates successful drug encapsulation without interference on surface construction.

\section{Figure 2}

Uptake of liposomes. Confocal images RCC-786 cells incubated with fluorescently tagged liposomes (TSLs) inside cells 2A) Combined bright field and red fluorescence view 2B) red fluorescence and 2C) bright field. Figure 2D: Z-stack images were taken at different heights and were compiled to form a 3-D image of the liposomes taken up by the cells.

\section{Figure 3}

Viability of 786-O renal cell carcinoma after treatment. Figure 3A: Comparison of individual and 2-part treatments at 24, 48, and 72 hours. Figure 3B: Comparison of FUS alone, TKI alone, TKI/TSL alone, and the combined treatment at 24, 48, 72, and 96 hours. Figure 3C: Comparison of treatment with FUS alone, TKI/TSL alone, TKI/TSL with FUS activation at 12 hour intervals from 24-96 hours. The TKI was administered at a concentration of $10 \mu \mathrm{M}$ in all experimental groups. Values are means \pm standard errors of the means of three independent experiments. ${ }^{*} \mathrm{p}<$ $0.05, * * \mathrm{p}<0.01, * * * \mathrm{p}<0.001$. 


\section{Figure 4}

Comparison of drug loaded thermosensitive and non-thermosensitive liposomes both with and without FUS on renal cancer cells at 24, 48, and 72 hours. The TKI was administered at a concentration of $10 \mu \mathrm{M}$ in all experimental groups. Values are means \pm standard errors of the means of three independent experiments. ${ }^{*} \mathrm{p}<0.05, * * \mathrm{p}<0.01$, ${ }^{* * *} \mathrm{p}<0.001$.

\section{Figure 5}

Comparison of water bath immersion at $43^{\circ}$ and FUS activation on renal cancer cell viability 24 , 48 , and 72 hours post treatment with TKI/TSLs. The TKI was administered at a concentration of $10 \mu \mathrm{M}$ in all experimental groups. Values are means \pm standard errors of the means of three independent experiments. ${ }^{* *} \mathrm{p}<0.01, * * * \mathrm{p}<0.001$.

\section{Figure 6}

Density plots of viable, early apoptotic, and late apoptotic/necrotic 786-O renal cancer cells 72 hours post treatment as measured by flow cytometry (Figures 5A-C). Comparison of the percentage of viable cells post treatment at 24, 48, and 72 hours (Figure 6D). The TKI was administered at a concentration of $10 \mu \mathrm{M}$ in all experimental groups.

\section{Figure 7}

Schematic depicting FUS activation of TKI-loaded, thermosensitive liposomes. FUS activation increases temperatures $\left(43^{\circ} \mathrm{C}\right.$ for 90 seconds) within the renal tumor allowing for targeted drug release and tumor cell death.

\section{Figure 8}

Linear time plot of renal cancer cell viability after treatment with FUS alone (triangles), TKI/TSL alone (circles), and the combined treatment (squares). The red dashed line, obtained by adding the amount of cell viability reduction achieved by each individual treatment, is a representation of the expected value of combined treatment if individual treatments interacted in a non-synergistic, additive manner. The difference in cellular viability seen between this theoretical plot and the plot of the combined treatment actually observed is a visual representation of synergy between FUS and TKI/TSLs. The TKI was administered at a concentration of $10 \mu \mathrm{M}$ in all experimental groups. 

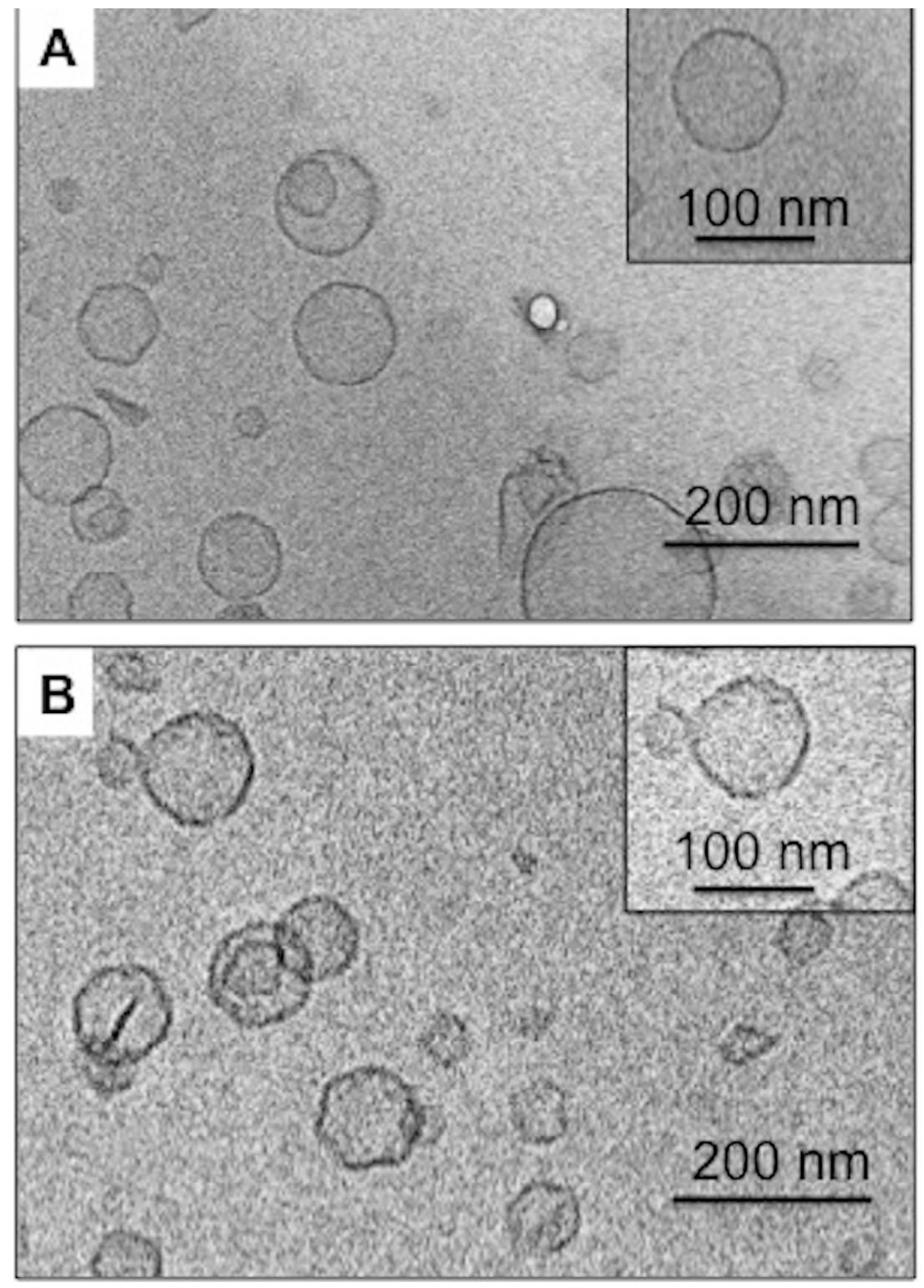


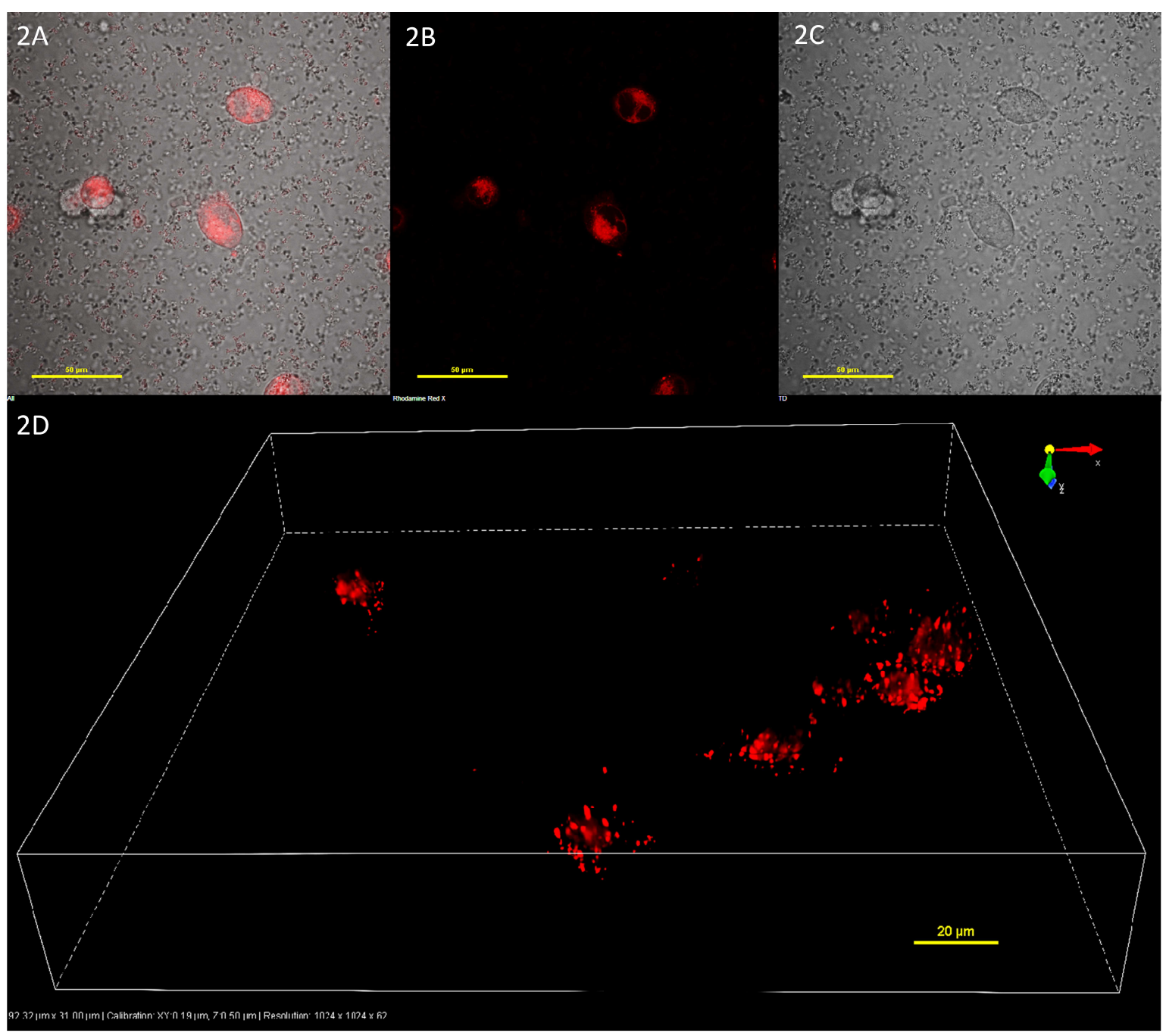




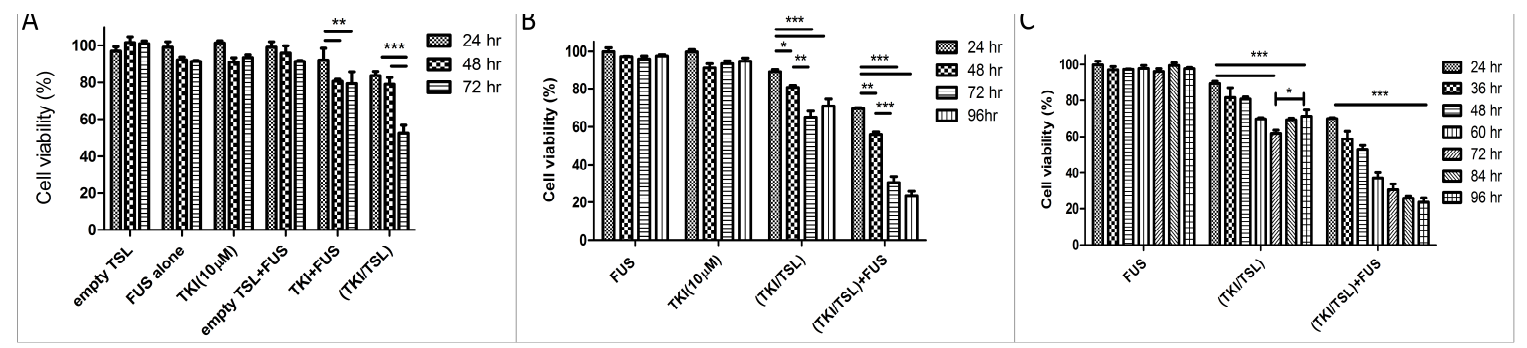




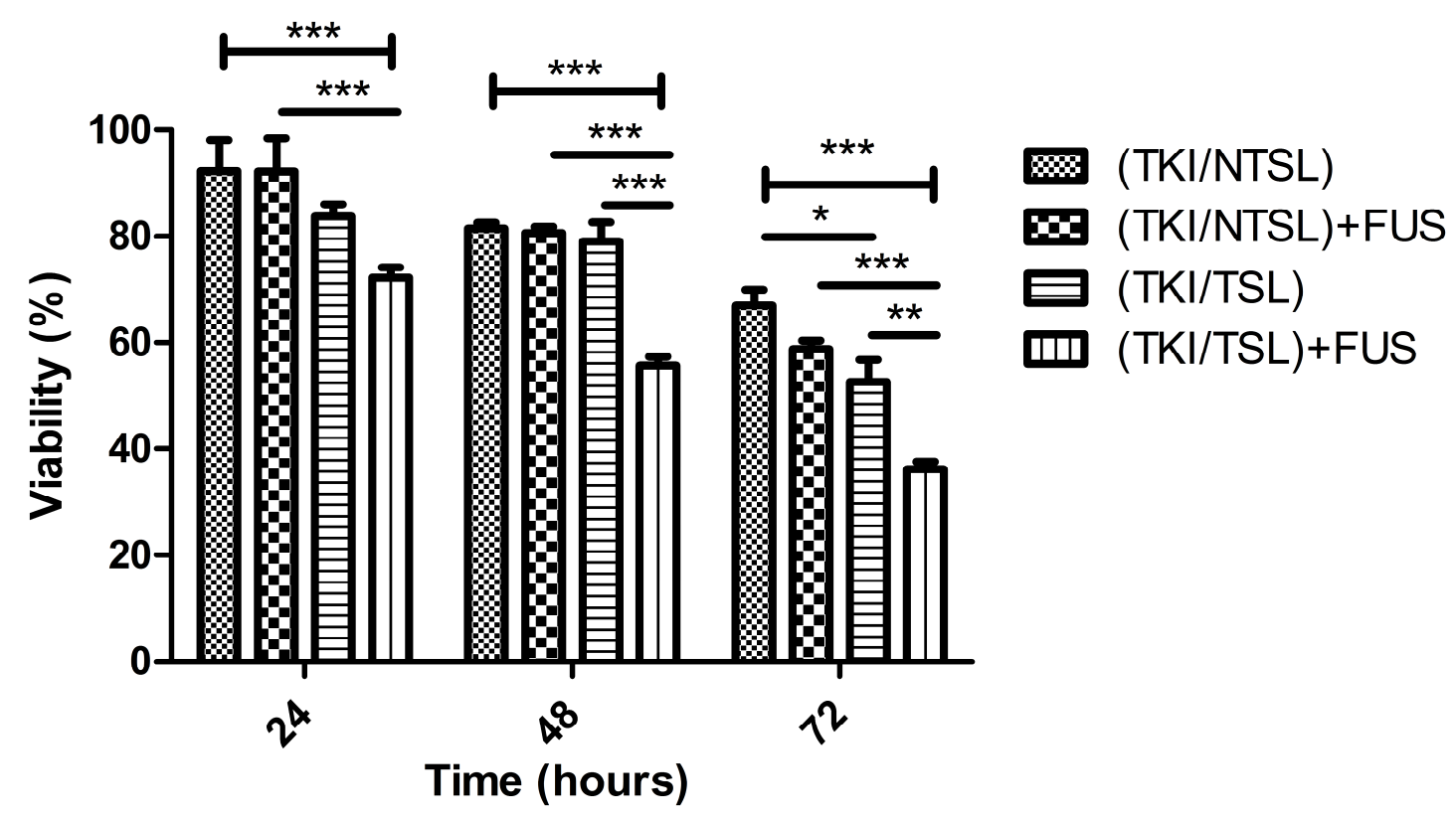




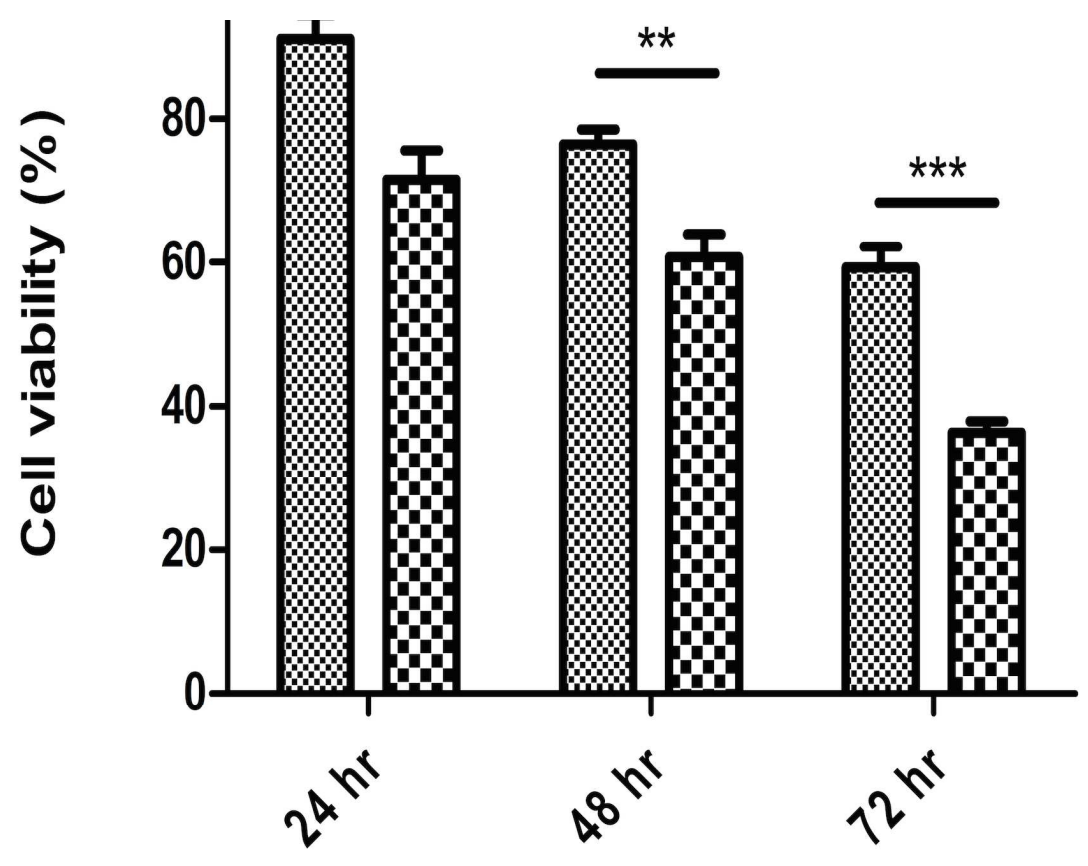




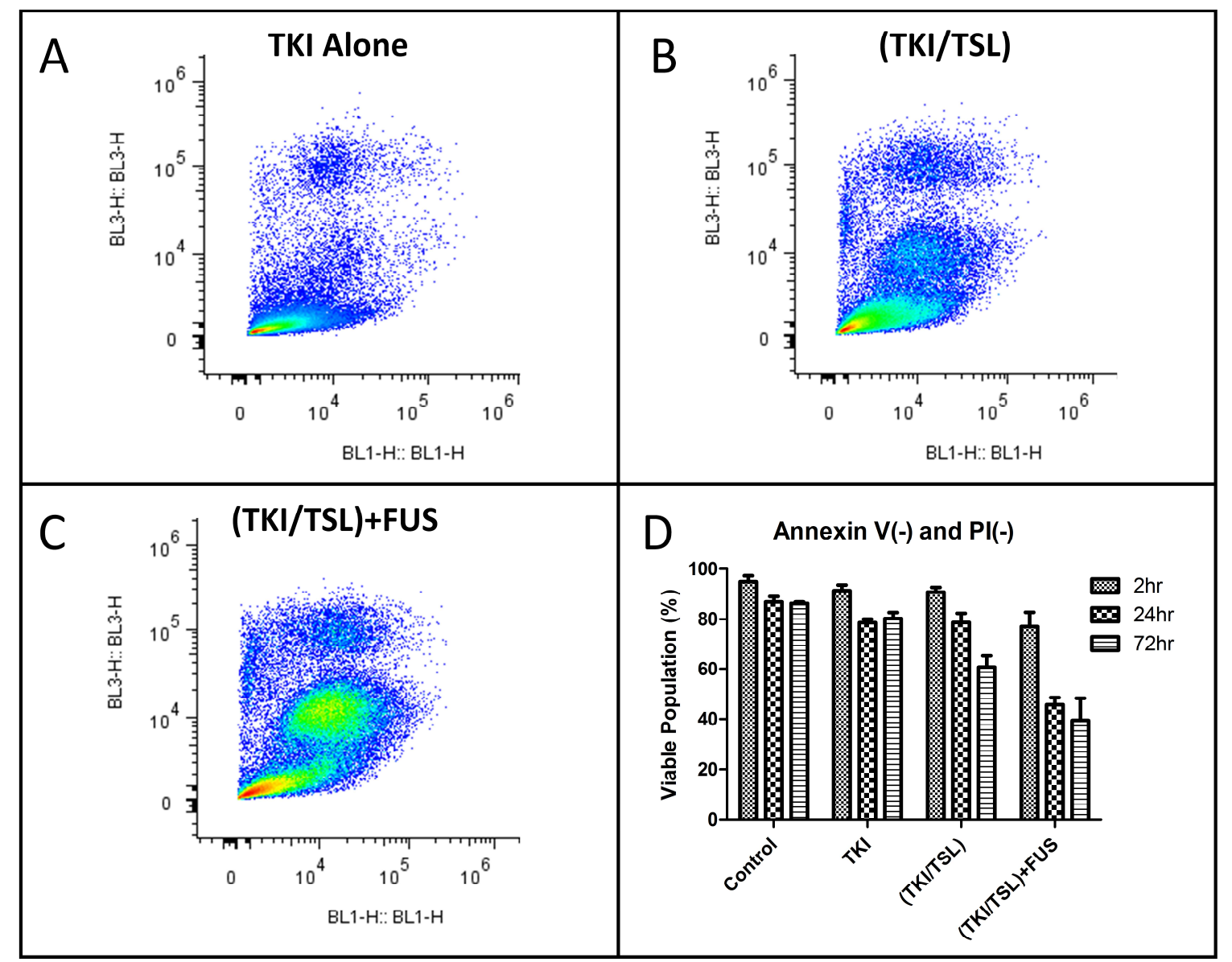



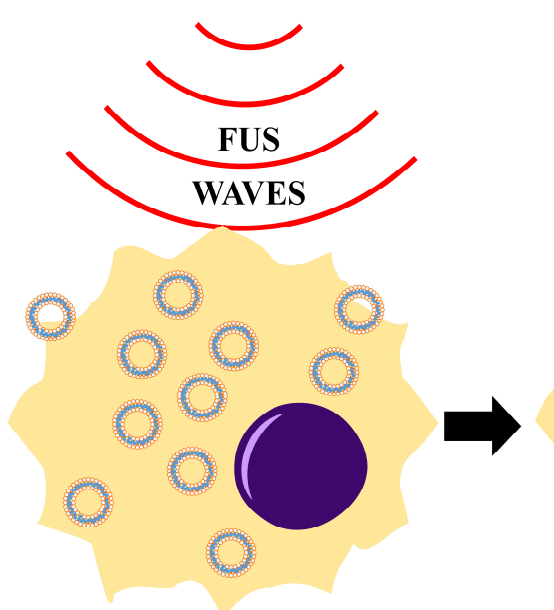

$37^{\circ} \mathrm{C}$

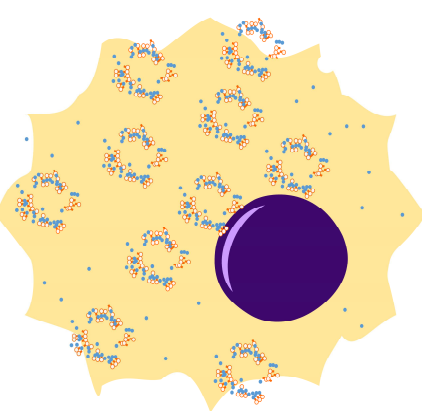

$43^{\circ} \mathrm{C}$

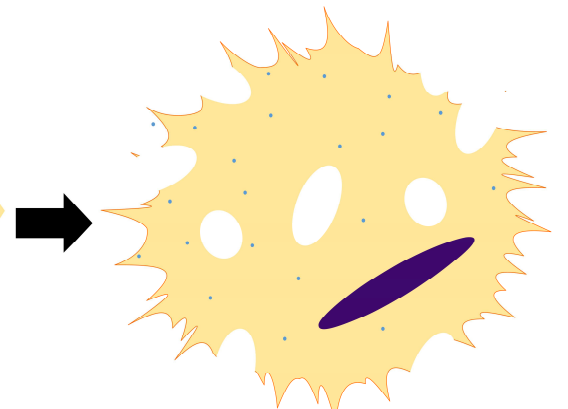

Apoptotic/Necrotic 


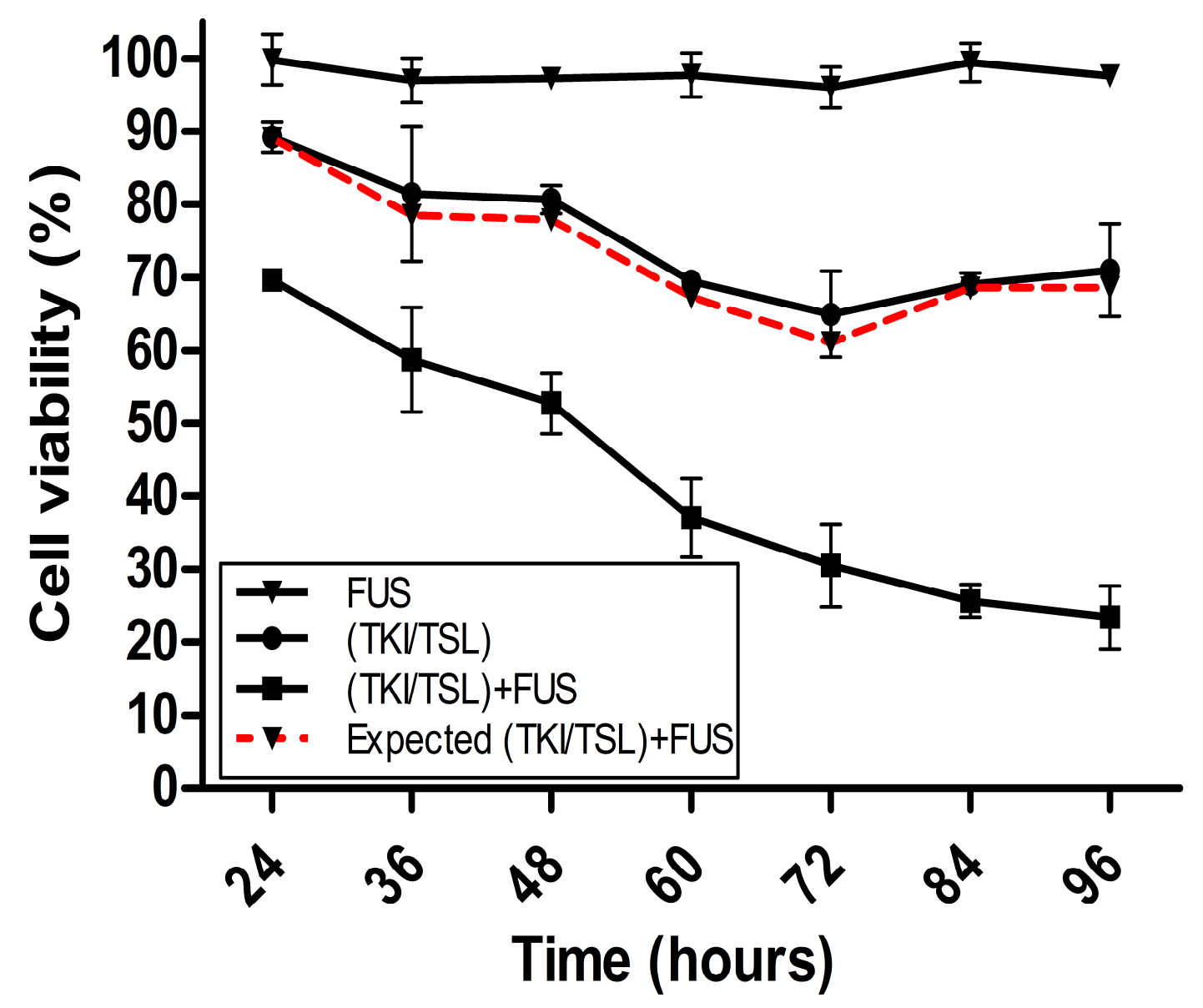

